\title{
Continuous Polling with Rerouting and Applications to Ferry Assisted Wireless LANs .
}

\author{
Veeraruna Kavitha \\ Maestro, INRIA, Sophia Antipolis, France and LIA, University of Avignon, France \\ kvoletiv@sophia.inria.fr
}

\begin{abstract}
In almost all studied continuous polling systems, the user leaves the system after his service is completed. There are interesting applications, in which the users demand a second service (or more). For example, in a ferry assisted wireless network, for every local data transfer the ferry has to collect the data from the source and then deliver the same to the sink. This type of application can be modeled by polling systems with rerouting. In polling systems with arrivals on a continuum (on a circle), a moving server attends the users as and when it encounters one. When rerouting is supported, after the service is completed, the users can reroute to a different point in the same circle to await another service. We obtain the performance of such a system under quite general conditions, via discretization approach. The results are applied to study a ferry assisted wireless local area network. Our results rely heavily on fixed point analysis of infinite dimensional operators.
\end{abstract}

\section{Keywords}

Continuous Polling systems, Rerouting, Virtual Workload, Pseudo Conservation Laws, Wireless LAN

\section{INTRODUCTION}

Polling systems are queueing systems, wherein a single server walks between a number of queues and attends to a queue as and when it meets one. Continuous polling systems are the ones in which arrivals can occur and the service can be derived, anywhere in a continuum. Most of these systems are studied under standard gated service. The server attends to the users immediately, as and when it encounters one. We then have a globally gated service $([19])$, wherein the server closes a global gate when it arrives say at 0 and tags all the users that arrived before the gate closure. Before retuning to 0 , it only serves the tagged users. In all these systems,

\footnotetext{
*This work was sponsored by The Indo-French Center for the Promotion of Advanced Research under CEFIPRA Project\# 4000-IT-1

Permission to make digital or hard copies of all or part of this work for personal or classroom use is granted without fee provided that copies are not made or distributed for profit or commercial advantage and that copies bear this notice and the full citation on the first page. To copy otherwise, to republish, to post on servers or to redistribute to lists, requires prior specific permission and/or a fee.

VALUETOOLS 2011, May 16-20, Paris, France

Copyright @ 2011 ICST 978-1-936968-09-1

DOI 10.4108/icst.valuetools.2011.245681
}

the user leaves the system after its service. But there can be applications, wherein the users require a second service or more. For example, in a Ferry assisted Wireless LAN (FWLAN), every local data transfer demands the ferry to provide its service at two independent locations in the network: 1) ferry collects data from the source; and 2) ferry deposits the collected data at the sink. In this paper, we study one such FWLAN, where the ferry facilitates communication not only between the base station (a gateway to the external world) and the network users, but also facilitates the inter user or local communication. Hence we will need a system that supports more than one service.

We consider a polling system in which the user after it's first service may be rerouted to a different point to obtain a second service. Using the discretization approach of our earlier paper [19], we obtain the stationary expected workload as the limit of the stationary expected workload of a discrete system. We discretize the continuous polling system in such a way that the Pseudo conservation laws of of Sidi et.al ([15]) for discrete polling systems with rerouting, are applicable. To the best of our knowledge this is the first paper which considers a continuous polling system with rerouting.

Related Work : Continuous polling systems were first introduced by Fuhrmann and Cooper [7], further explored by Coffman and Gilbert [3, 4] and Kroese and Schmidt [11, 8, $9,10]$ in a series of works. Stability results are available in $[12,14,13]$. The continuous systems are usually analyzed under simplified conditions, which we refer as 'symmetric conditions': Poisson arrivals and every arrival picks up a uniformly-distributed landing site on the circle while the server is moving at a constant speed in a fixed direction. Further, the service times are identically distributed.

Snowplowing systems generalize many of the above assumptions and study a more general continuous system. For example, the incoming work-flow to the system is taken to be a general Lévy random measure and the walking times are assumed to be random (see for example $[5,6]$ ).

In literature, continuous polling systems have been usually analyzed with simple gated/exhaustive service. In [19] we study continuous polling systems which offer either globally gated service or gated service or a mixture of the two services or elevator service, without requiring symmetry. Even in this paper, we do not assume the symmetric assumptions. 
Polling systems are widely used for modeling and analysis in various scenarios, like in communication systems, computer hardware and software, road traffic control. For example, in [23, 22, 21], authors analyze FWLANs. Apart from FWLANs, there are many other applications, in which customers may require more than one service. Various such applications are mentioned in [15], for example: 1) selectiverepeat ARQ protocol used to recover from transmission errors; 2) computer system where a single processor often has the responsibility of performing many distinct tasks, such as computation, sending information to memory, retrieve information. The results of this paper can be applied in the aforementioned applications, whenever the arrivals are on a continuum. This paper (in contrast to [15]) supports at maximum one rerouting, however in the extension of this work ([20]), the polling systems support many but finite number of reroutings and hence can model almost all the (continuous versions of the) applications mentioned in [15].

The system, the model and the notations of the paper are introduced in section 2 . In the same section we also brief upon the main result of the paper, Theorem 1 . We discuss the discretization in section 3 while the proof of the Theorem is obtained in sections 4 and 5 . Ferry based wireless LAN is analyzed in section 6. Some proofs are in the Appendix.

\section{CONTINUOUS POLLING SYSTEMS}

We consider a continuous polling system, where the server moves continuously on a circle $\mathcal{C}$ of radius $R$ with speed $\alpha$ and stops at a point only when it finds a user with request. The external arrival process is modeled by a Poisson process with intensity $\lambda$ and every external/new arrival is associated with two marks, the position $Q \in \mathcal{C}$ distributed as $P_{Q}$ and the service times $B_{e}$. The service times in general can depend upon the position $Q$ of the arrival. Let $b_{e}(q) b_{e}^{(2)}(q)$ represent the conditional first two moments of the service time $B_{e}$ conditioned on the event that $Q$ is at $q$. The service times of different users are independent of each other.

Every user is serviced the first time the server encounters him on the circle. After his service is completed, the user is either rerouted, independent of all the previous events, to a new point $q$ on the circle with probability $\epsilon P_{R}(d q)$ with $P_{R}(d q)=f_{R}(q) d q$ or exists the system with $1-\epsilon$ probability. So the rerouting probabilities are independent of $Q$, the position at which the first service is obtained. The rerouted users are serviced the next time the server meets them and then they exit. So no rerouting after rerouting.

The users are rerouted independent of the previous happenings, however their service requirements for the second service can again depend upon the position of the rerouted point (which is distributed as $\left.P_{R}\right)$. Let $b_{r}(q), b_{r}^{(2)}(q)$ represent the conditional first and second moments of the service time, $B_{r}$, demanded by the rerouted users given that the rerouted arrival, $R$, is at point $q$. Let $\bar{b}_{e}:=E_{Q}\left[b_{e}(Q)\right]$, $\bar{b}_{r}:=E_{R}\left[b_{r}(R)\right]$ (the expectations are respectively with respect to $P_{Q}$ and $P_{R}$ ) represent the unconditional moments. Similarly define, $\bar{b}_{e}^{(2)}:=E_{Q}\left[b_{e}^{(2)}(Q)\right]$ and $\bar{b}_{r}^{(2)}:=E_{R}\left[b_{r}^{(2)}(R)\right]$.

Notations : The variables like $b_{e}, b_{r}, f_{Q}, f_{R}, \tau$ represent nonnegative functions on interval $[0,|\mathcal{C}|](|\mathcal{C}|$ is length of $\mathcal{C})$ while terms like $b_{e}(q)$ or $\tau(q)$ represent their value at a point $q \in[0,|\mathcal{C}|]$. The bar of the same variable, for example $\bar{b}_{e}$, represents the average w.r.t. to the corresponding position distribution. Similarly variables like $\tau^{(2)}, \tilde{\tau}^{(2)}$ etc. are functions on $[0,|\mathcal{C}|] \times[0,|\mathcal{C}|]$ while $\tau^{(2)}\left(q, q^{\prime}\right)$ represents the function value at $\left(q, q^{\prime}\right)$. The expectations are represented by symbol $E$ and these expectations are either with respect to $R, Q$ or stationary measure of the process. In situations of ambiguity we suffix $E$ with either $R$ or $Q . E^{0}$ represents expectation under Palm stationarity. Define the following:

$$
\begin{aligned}
\rho_{e}([a, c]) & :=\lambda \int_{a}^{c} b_{e}(q) f_{Q}(q) d q, \quad \rho_{e}:=\rho_{e}([0,|\mathcal{C}|]), \\
\rho_{r}([a, c]) & :=\lambda \int_{a}^{c} b_{r}(q) f_{R}(q) d q, \quad \rho_{r}:=\rho_{r}([0,|\mathcal{C}|]), \\
\rho([a, c]) & :=\rho_{e}([a, c])+\epsilon \rho_{r}([a, c]) \text { and } \rho=\rho_{e}+\epsilon \rho_{r} .
\end{aligned}
$$

\subsection{Main Result}

We assume that the continuous polling system with rerouting is in stationary ergodic regime. Virtual workload of a polling system is the sum workload of all the waiting users. We obtain the stationary expected virtual workload as a limit of the same for a 'certain' set of the discrete polling systems.

THEOREM 1. Assume that $b_{e}, b_{r}, f_{R}$ and $f_{Q}$ are continuous. Then there exists a threshold $\lambda_{0}$ (given by equation (26) in Appendix) and for all Poisson arrivals at rates $\lambda<\lambda_{0}$, the expected stationary virtual workload for the continuous polling system with rerouting, $V_{r r t}$, is given by:

$$
\begin{aligned}
& V_{r r t}=\rho \frac{\lambda \bar{b}_{e}^{(2)}+\epsilon \lambda \bar{b}_{r}^{(2)}}{2(1-\rho)}+\frac{\rho \epsilon \lambda \bar{b}_{r} \bar{b}_{e}}{1-\rho}+\frac{\rho|\mathcal{C}| \alpha^{-1}}{2}+\frac{\lambda \alpha^{-1}}{1-\rho} \\
& \int_{0}^{|\mathcal{C}|} \int_{0}^{|\mathcal{C}|}\left(b_{e}\left(q^{\prime}\right)+\epsilon \bar{b}_{r}\right)\left(\hat{\rho}(q)-\hat{\rho}\left(q^{\prime}\right)+1_{\left\{q<q^{\prime}\right\}} \rho\right) f_{Q}\left(q^{\prime}\right) d q^{\prime} d q \\
& +\frac{\epsilon \lambda \alpha^{-1}}{1-\rho} \int_{0}^{|\mathcal{C}|} \int_{0}^{|\mathcal{C}|} b_{r}\left(q^{\prime}\right)\left(q^{\prime}-q+|\mathcal{C}| 1_{\left\{q>q^{\prime}\right\}}\right) f_{R}\left(q^{\prime}\right) d q^{\prime} f_{Q}(q) d q, \\
& \text { where } \hat{\rho}(q):=\rho([0, q]), \rho=\rho([0,|\mathcal{C}|]) .
\end{aligned}
$$

\section{$\underline{\text { Special Cases: }}$}

1) Under symmetric conditions: Uniform arrivals, i.e., $P_{Q}$, $P_{R}$ are uniform. The moments $b_{e}(q), b_{e}^{(2)}(q) b_{r}(q)$ and $b_{r}^{(2)}(q)$ are equal at all $q$ and equal $\bar{b}_{e}, \bar{b}_{e}^{(2)} \bar{b}_{r}$ and $\bar{b}_{r}^{(2)}$ respectively. Then $\hat{\rho}(q)=\rho q /|\mathcal{C}|$ and (1) simplifies to (with $\rho=\bar{b}_{e}+\epsilon \bar{b}_{r}$ ):

$V_{r r t}^{\text {sym }}=\rho \frac{\lambda \bar{b}_{e}^{(2)}+\epsilon \lambda \bar{b}_{r}^{(2)}}{2(1-\rho)}+\frac{\rho \epsilon \lambda \bar{b}_{r} \bar{b}_{e}}{1-\rho}+\frac{|\mathcal{C}| \alpha^{-1}\left(\rho+\epsilon \lambda \bar{b}_{r}\right)}{2(1-\rho)}$.

2) Gated/Exhaustive polling system: under symmetric conditions (case 1) is analyzed in [11]. By [11, Theorem 5.1], the stationary expected number of waiting users (with $|\mathcal{C}|=1$ ):

$$
E[\bar{N}]_{e}=\lambda \bar{b}_{e}+\frac{\lambda\left(\alpha^{-1}+\lambda \bar{b}_{e}^{(2)}\right)}{2\left(1-\lambda \bar{b}_{e}\right)} .
$$

They also considered the user under service. Excluding it,

$$
E[N]_{e}=\frac{\lambda\left(\alpha^{-1}+\lambda \bar{b}_{e}^{(2)}\right)}{2\left(1-\lambda \bar{b}_{e}\right)} .
$$


By Wald's lemma the expected virtual workload equals :

$$
V_{e}^{s y m}=\bar{b}_{e} E[N]_{e}=\frac{\lambda \bar{b}_{e}\left(\alpha^{-1}+\lambda \bar{b}_{e}^{(2)}\right)}{2\left(1-\lambda \bar{b}_{e}\right)} .
$$

This matches with (2) when $|\mathcal{C}|=1, \epsilon=0$ (no rerouting).

3) Gated/Exhaustive polling system under general conditions: In [19] we studied a mixed continuous polling system which supports gated as well as globally gated service users. We derived the formula for the stationary expected virtual workload. From Theorem 1, [19] the virtual workload for pure gated service is given by (with $p_{g g}=0=1-p_{g}$ ):

$$
V_{e}=\lambda \bar{b} \frac{\lambda \bar{b}^{(2)}}{2(1-\lambda \bar{b})}+\frac{\lambda \bar{b}|\mathcal{C}| \alpha^{-1}}{2}+\frac{|\mathcal{C}| \alpha^{-1}}{2(1-\lambda \bar{b})}\left(\lambda^{2} \bar{b}^{2}\right) .
$$

The first integral in the formula (1) with no rerouting $(\epsilon=0)$ but under general conditions equals ${ }^{1}$ :

$$
\begin{aligned}
& \int_{0}^{|\mathcal{C}|} \int_{0}^{|\mathcal{C}|} b_{e}\left(q^{\prime}\right)\left(\hat{b}_{e}(q)-\hat{b}_{e}\left(q^{\prime}\right)+1_{\left\{q<q^{\prime}\right\}} \bar{b}_{e}\right) f_{Q}\left(q^{\prime}\right) d q^{\prime} d q \\
& =\bar{b}_{e} \int_{0}^{|\mathcal{C}|} \hat{b}_{e}(q) d q-|\mathcal{C}| E_{Q}\left[b_{e}(Q) \hat{b}_{e}(Q)\right]+\bar{b}_{e} \int_{0}^{|\mathcal{C}|}\left(\bar{b}_{e}-\hat{b}_{e}(q)\right) d q \\
& =\bar{b}_{e}^{2} \frac{|\mathcal{C}|}{2}, \quad \text { where } \hat{b}_{e}(q):=\int_{0}^{q} b_{e}(y) f_{Q}(y) d y .
\end{aligned}
$$

Thus for pure gated service formula (1) simplifies to:

$$
V_{r r t}=\rho \frac{\lambda \bar{b}_{e}^{(2)}}{2(1-\rho)}+\frac{\rho|\mathcal{C}| \alpha^{-1}}{2}+\frac{\lambda^{2} \alpha^{-1}}{1-\rho}+\frac{|\mathcal{C}|}{2} \frac{\lambda^{2} \bar{b}_{e}^{2} \alpha^{-1}}{1-\rho}
$$

which matches with (4).

The main aim of this paper is to obtain the proof of Theorem 1. We later apply the result, formula (1), to analyze a Ferry aided Wireless LAN in section 6 . The proof is obtained in the following 3 major steps (as done in [19]). The methodology of the proof might be same as that in ([19]), it however requires significant changes to take care of rerouting. In this paper, we only present those parts of the proof which are different from that in ([19]) and refer to [19] for the common parts.

1)Discretization: Continuous polling system with rerouting is converted to an appropriate discrete polling system with rerouting in section 3 , for which the Pseudo conservation laws and hence the stationary expected virtual workload is known (see [15]).

2)Fixed point equations: We express the stationary moments of the time to reach a point (for exact details refer to section 4) on the circumference starting from some

$$
\begin{aligned}
& { }^{1} \text { By interchanging the order of the two integrals, } \\
& \begin{aligned}
E\left[b_{e}(Q) \hat{b}_{e}(Q)\right]=\int_{0}^{|\mathcal{C}|}\left(\int_{0}^{q^{\prime}} b_{e}(q) f_{Q}(q) d q\right) b_{e}\left(q^{\prime}\right) f_{Q}\left(q^{\prime}\right) d q^{\prime} \\
=\int_{0}^{|\mathcal{C}|}\left(\int_{q}^{|\mathcal{C}|} b_{e}\left(q^{\prime}\right) f_{Q}\left(q^{\prime}\right) d q^{\prime}\right) b_{e}(q) f_{Q}(q) d q \\
=\int_{0}^{|\mathcal{C}|}\left(\bar{b}_{e}-\int_{0}^{q} b_{e}\left(q^{\prime}\right) f_{Q}\left(q^{\prime}\right) d q^{\prime}\right) b_{e}(q) f_{Q}(q) d q
\end{aligned}
\end{aligned}
$$

and so, $E\left[b_{e}(Q) \hat{b}_{e}(Q)\right]=\bar{b}_{e}^{2} / 2$. fixed point, 0 , as a fixed point of a linear operator in section 4. We obtain a common linear operator $((\mathcal{F}, \Theta)$ defined in section 4), actually an affine linear map, which is further parametrized by $\sigma$, the number of discretization levels. The fixed point of the common operator at $\sigma<\infty$ gives the required stationary moments for the discrete system while that at $\sigma=\infty$ corresponds to the continuous system. We show the continuity of these fixed points as $\sigma \rightarrow \infty$ via contraction mapping theorem and hence show that the stationary moments of the discrete system converge to that of the continuous system.

3)Alternate expression for Virtual Workload: We express the stationary expected virtual workload in terms of the stationary moments of Step 2. Note, that this common expression cannot be computed easily and is used only for the proof. We again show the continuity of this common expression as $\sigma \rightarrow \infty$ and hence the convergence of the stationary expected virtual workload of the discrete system to that of the continuous system in section 5 .

\section{DISCRETIZATION}

For each integer $\sigma$, we construct a discrete polling system with $2 \sigma$ queues, half of which serve only external arrivals while the rest half serve only the rerouted users. The circumference $|\mathcal{C}|$ is divided to $\sigma$ equal segments $\left\{I_{i}\right\}_{i=1}^{\sigma}$ with $I_{1}=[0, \mathcal{C} / \sigma]$. External Arrivals are as in the continuous system. Users arriving in area $I_{i}$ are treated as though arriving in queue numbered $2 i-1$ (for rerouted users) or $2 i$ (for external users). For every $i$ the server stops upon reaching,

$$
i^{\sigma}:=(i-1) \frac{|\mathcal{C}|}{\sigma},
$$

the starting point of $I_{i}$ and serves all the users of $I_{i}$ before moving further. It first attends the "rerouted" queue using gated service, i.e., serves all those rerouted users of $I_{i}$ that arrived before the server reached $i^{\sigma}$. After that, the server attends the "external" queue once again using gated service, i.e., it serves all the external arrivals of $I_{i}$ that arrived before the server completed with the rerouted queue of $I_{i}$. We refer these as rerouted queues and external queues.

Within a queue, the server attends the users using a special order which we call as arrival position order. In this special order, the users within a queue are served in the order of their distance from the stop $i^{\sigma}$ of the server, i.e., the user at minimum distance is served first. So, the users are almost served in the same order as done in a continuous system. The main difference $\mathrm{b} / \mathrm{w}$ the continuous system and the discretized system is that some of the users are postponed to the next cycle in the discretized system. This is mainly because of the combined effect of discretization and the gated service. But we will see that the effect of these difference users reduces to zero as $\sigma$ tends to $\infty$.

The external arrivals after their first service (at one of the even numbered queues) are either rerouted (to an odd numbered queue) or exit according to the following probabilities:

$$
\begin{aligned}
P_{2 i, 2 j-1} & =\operatorname{Prob}\left(\text { User arrived in }\left[i^{\sigma},(i+1)^{\sigma}\right]\right. \\
& \left.\quad \text { is rerouted to }\left[j^{\sigma},(j+1)^{\sigma}\right]\right) \\
& =\epsilon P_{R}\left(I_{j}\right) \quad \text { by independence and } \\
P_{2 i, 2 j}= & P_{2 i-1,2 j}=P_{2 i-1,2 j-1}=0 \text { for all } i, j .
\end{aligned}
$$


Poisson arrivals into the system occur with intensity $\lambda$ and land in one of the even queues: for example the arrivals in area $I_{i}$ form the external arrivals to queue numbered $2 i$. Thus, the rate of external arrivals at different queues are:

$$
\lambda_{2 i}=\lambda P_{Q}\left(I_{i}\right) \text { and } \lambda_{2 i-1}=0 \text { for all } i \leq \sigma .
$$

An user at external (rerouted) queue $2 i(2 i-1)$, demands service $B_{e}\left(B_{r}\right)$ and this service is conditioned on the event that the arrival is in $I_{i}$. Thus, the service time moments at different queues are:

$$
\begin{array}{rlrl}
b_{2 i} & =\frac{E\left[B_{e} 1_{\left\{Q \in I_{i}\right\}}\right]}{P_{Q}\left(I_{i}\right)}, & b_{2 i}^{(2)} & =\frac{E\left[B_{e}^{2} 1_{\left\{Q \in I_{i}\right\}}\right]}{P_{Q}\left(I_{i}\right)}, \\
b_{2 i-1} & =\frac{E\left[B_{r} 1_{\left\{R \in I_{i}\right\}}\right]}{P_{R}\left(I_{i}\right)} \text { and } b_{2 i-1}^{(2)}=\frac{E\left[B_{r}^{2} 1_{\left\{R \in I_{i}\right\}}\right.}{P_{R}\left(I_{i}\right)} .
\end{array}
$$

The overall arrival rates $\gamma_{i}$ (resulting after rerouting) can be calculated solving equation (2.1), [15] as:

$$
\begin{aligned}
\gamma_{2 i} & =\lambda_{2 i}+\sum_{j}^{2 \sigma} \gamma_{j} P_{j, 2 i} \Longrightarrow \gamma_{2 i}=\lambda_{2 i} \text { for all } i \text { and so } \\
\gamma_{2 i-1} & =\lambda_{2 i-1}+\sum_{j=1}^{2 \sigma} \gamma_{j} P_{j, 2 i-1} \Longrightarrow \gamma_{2 i-1}=\epsilon \lambda P_{R}\left(I_{i}\right) .
\end{aligned}
$$

The overall service time requirements resulting from the first and the possible second service, $\tilde{b}$, can be calculated as below (solving equations 2.3 and 2.4 of [15]):

$$
\begin{array}{lll}
\tilde{b}_{2 i}=b_{2 i}+\epsilon \bar{b}_{r} & \tilde{b}_{2 i}^{(2)}=b_{2 i}^{(2)}+2 \epsilon b_{2 i} \bar{b}_{r}+\epsilon \bar{b}_{r}^{(2)} \\
\tilde{b}_{2 i-1}=b_{2 i-1} & \tilde{b}_{2 i-1}^{(2)}=b_{2 i-1}^{(2)} .
\end{array}
$$

In the above, $\left\{\lambda_{2 i-1}\right\}_{i=1}^{\sigma},\left\{b_{2 i-1}\right\},\left\{b_{2 i-1}^{(2)}\right\}$ respectively represent the arrival rates, the first and the second moments of the service times at the rerouted queues while the corresponding remaining represent the same for external queues.

Define, $\rho_{i}:=\gamma_{i} b_{i}$ and $\rho=\sum_{i} \rho_{i}=\lambda \bar{b}_{e}+\epsilon \lambda \bar{b}_{r}$.

Note that $\rho$ is same for all $\sigma$. It represents the total work load in the system and the discrete system is stable only when $\rho<1$ ([15]). This condition is guaranteed because: continuous polling system is assumed to be stable, so the stationary moments of Theorem 2 (given in section 4) exist and by the same theorem it is possible if and only if $\rho<1$.

Expected Stationary Workload for discrete system: We thus have a $2 \sigma$ stable polling system with even numbered queues experiencing the gated service with external arrivals and the odd numbered queues also experiencing the gated service but with only rerouted users. Further we have fixed walking times between queues, that between the queues of the same stop is zero, $r_{2 i-1}=0$ and $r_{2 i}=|\mathcal{C}| \alpha^{-1} / \sigma$. This type of a discrete polling system with rerouting is considered in [15]. By Pseudo Conservation Laws of [15] the expected stationary virtual workload of the $\sigma$-polling model with rerouting is (from equation (6.4) of [15] after removing the zero terms):

$$
\begin{aligned}
V_{r r t}^{\sigma}= & \frac{\sum_{j=1}^{\sigma} \lambda_{2 j} \tilde{b}_{2 j}^{(2)}}{2(1-\rho)}-\frac{\lambda \bar{b}_{e}^{(2)}+\epsilon \lambda \bar{b}_{r}^{(2)}}{2}-\epsilon \lambda \bar{b}_{r} \bar{b}_{e} \\
& +\frac{\rho|\mathcal{C}| \alpha^{-1}}{2}+\frac{1}{1-\rho} \sum_{i=1}^{\sigma} \frac{|\mathcal{C}| \alpha^{-1}}{\sigma} \sum_{j=1}^{\sigma} \lambda_{2 j} \tilde{b}_{2 j} \sum_{k=2 j}^{2 i} \rho_{k} \\
& +\frac{1}{1-\rho} \sum_{i=1}^{\sigma} \sum_{j=1}^{\sigma} \gamma_{2 i} \epsilon P_{R}\left(I_{j}\right) \tilde{b}_{2 j-1} \sum_{k=2 i}^{2 j-2} r_{k} .
\end{aligned}
$$

The results of $[16,17,18,15]$ are valid for any work conserving order at each queue and hence the results are also valid for our arrival position order.

Limit of the Virtual Workload: As obtained in the proof of Lemma 1, which is available in [19], one can find the limit (as $\sigma \rightarrow \infty$ ) of the above workload $V_{r r t}^{\sigma}$ via Riemann sums. The first 4 terms of equation (5) are independent of $\sigma$ and simplify as the first three terms of (1). Note that

$$
\lambda_{2 j} \tilde{b}_{2 j}=\lambda E\left[\left(B_{e}+\epsilon \bar{b}_{r}\right) 1_{\left\{Q \in I_{j}\right\}}\right]=\lambda E_{Q}\left[\left(b_{e}(Q)+\epsilon \bar{b}_{r}\right) 1_{\left\{Q \in I_{j}\right\}}\right] .
$$

Thus the fifth term of (5) can be approximated by:

$$
\begin{aligned}
& \frac{1}{1-\rho} \sum_{i=1}^{\sigma} \frac{|\mathcal{C}| \alpha^{-1}}{\sigma} \sum_{j=1}^{\sigma} \lambda_{2 j} \tilde{b}_{2 j} \sum_{k=2 j}^{2 i} \rho_{k} \\
& \quad \approx \frac{\lambda \alpha^{-1}}{1-\rho} \sum_{i=1}^{\sigma} \frac{|\mathcal{C}|}{\sigma} \sum_{j=1}^{\sigma}\left(b_{e}\left(j^{\sigma}\right)+\epsilon \bar{b}_{r}\right) f_{Q}\left(j^{\sigma}\right) \frac{|\mathcal{C}|}{\sigma} \sum_{k=2 j}^{2 i} \rho_{k} .
\end{aligned}
$$

The circular summation (see [15]), approximately equals:

$$
\begin{aligned}
& \sum_{k=2 j}^{2 i} \rho_{k}=1_{\{i \geq j\}} \sum_{k=2 j}^{2 i} \rho_{k}+1_{\{i<j\}}\left(\sum_{k=2 j}^{2 \sigma} \rho_{k}+\sum_{k=1}^{2 i} \rho_{k}\right) \\
& \approx 1_{\{i \geq j\}} \rho\left(\left[j^{\sigma}, i^{\sigma}\right]\right)+1_{\{i<j\}}\left[\rho-\rho\left(\left[i^{\sigma}, j^{\sigma}\right]\right)\right] \\
& =\rho\left(\left[0, i^{\sigma}\right]\right)-\rho\left(\left[0, j^{\sigma}\right]\right)+1_{\{i<j\}} \rho=\hat{\rho}\left(i^{\sigma}\right)-\hat{\rho}\left(j^{\sigma}\right)+1_{\{i<j\}} \rho .
\end{aligned}
$$

The error in the two approximations can be shown to converge to zero (as done in [19]) by Bounded Convergence Theorem. By substituting the above approximation in (6) we obtain a Riemann sum, which converges and hence the fifth term of (5) converges to:

$$
\begin{array}{r}
\frac{\lambda \alpha^{-1}}{1-\rho} \int_{0}^{|\mathcal{C}|} \int_{0}^{|\mathcal{C}|}\left(b_{e}\left(q^{\prime}\right)+\epsilon \bar{b}_{r}\right)\left(\hat{\rho}(q)-\hat{\rho}\left(q^{\prime}\right)+1_{\left\{q<q^{\prime}\right\}} \rho\right) \\
f_{Q}\left(q^{\prime}\right) d q^{\prime} d q .
\end{array}
$$

In a similar way the sixth term of (5) is approximated by:

$$
\begin{aligned}
\frac{\epsilon \lambda \alpha^{-1}}{1-\rho} \sum_{i=1}^{\sigma} f_{Q}\left(i^{\sigma}\right) \frac{|\mathcal{C}|}{\sigma} \sum_{j=1}^{\sigma} b_{r}\left(j^{\sigma}\right) f_{R}\left(j^{\sigma}\right) & \\
& \left(\frac{j-i}{\sigma}|\mathcal{C}| 1_{\{i<j\}}+\frac{\sigma-(i-j)}{\sigma}|\mathcal{C}| 1_{\{i \geq j\}}\right)
\end{aligned}
$$

and hence it converges to (note $(i-1)|\mathcal{C}| / \sigma=i^{\sigma}$ ):

$\frac{\epsilon \lambda \alpha^{-1}}{1-\rho} \int_{0}^{|\mathcal{C}|} \int_{0}^{|\mathcal{C}|} b_{r}\left(q^{\prime}\right)\left(q^{\prime}-q+|\mathcal{C}| 1_{\left\{q>q^{\prime}\right\}}\right) f_{R}\left(q^{\prime}\right) d q^{\prime} f_{Q}(q) d q$.

Thus (5) converges to (1).

\section{FIXED POINT EQUATIONS}

Let 0 be any arbitrary point of the circumference, $\mathcal{C}$. We call the time period between two successful visits of the server at point 0 as cycle. Let $\phi_{n}^{\sigma}(q)$ represent the time at which the server starts the service of the external queue (even numbered queue with only external arrivals) in the $n^{\text {th }}$ cycle, to which the point $q$ belongs. In case of the continuous system, this corresponds to the instance the server touches the point $q$ in the $n^{\text {th }}$ cycle. Let $T_{n}^{\sigma}(q):=\phi_{n}^{\sigma}(q)-\phi_{n}^{\sigma}(0)$, represent the time taken by the server to travel starting from 0 while serving all the users (with requests) on the way till the time the service of the external queue, to which point $q$ belongs, begins in the $n^{\text {th }}$ cycle. Let $\mathcal{T}_{e}([a, c], T)$ represent the total workload of the Poisson (external) arrivals that arrived 
in strip $[a, c] \subset[0,|\mathcal{C}|]$, such that a user at point $q \in[a, c]$ can arrive during a period of time $T(q)$. Let $\mathcal{T}_{r}([a, c], T)$ represent the workload (in the second service) due to that fraction of the external arrivals, $\mathcal{T}_{e}([0,|\mathcal{C}|], T)$, which were rerouted to the strip $[a, c]$ after their first service. Let

$$
C_{n}^{\sigma}(q) \triangleq \phi_{n+1}^{\sigma}(q)-\phi_{n}^{\sigma}(q)=T_{n}^{\sigma}(q)+T_{n-1}^{\sigma}(|\mathcal{C}|)-T_{n-1}^{\sigma}(q)
$$

represent the cycle time with respect to point $q$.

For every $q$, let $\delta^{\sigma}(q)$ represent the point standing at which the server attends $q$ (recall $\left.i^{\sigma}=(i-1)|\mathcal{C}| / \sigma\right)$ :

$\delta^{\sigma}(q)= \begin{cases}\sum_{i=1}^{\sigma} i^{\sigma} 1_{\left\{q \in I_{i}\right\}} ; I_{i}:=\left[i^{\sigma},(i+1)^{\sigma}\right) & \text { if } \quad \sigma<\infty \\ \text { if } \quad \sigma=\infty .\end{cases}$

With the above definitions (note at $\sigma=\infty,|\mathcal{C}| / \sigma=0$ ):

$$
\begin{gathered}
T_{n}^{\sigma}(q)=T_{n}^{\sigma}\left(\delta^{\sigma}(q)\right)=\delta^{\sigma}(q) \alpha^{-1}+\mathcal{T}_{e}\left(\left[0, \delta^{\sigma}(q)\right], C_{n-1}^{\sigma}\right) \\
+\mathcal{T}_{r}\left(\left[\frac{|\mathcal{C}|}{\sigma}, \delta^{\sigma}(q)+\frac{|\mathcal{C}|}{\sigma}\right], C_{n-2}^{\sigma}\right)
\end{gathered}
$$

This is the most important equation and is derived as explained below. In (8), the first term represents the time taken to travel the distance. The second term represents the time taken to complete service of the external arrivals at positions placed before $\delta^{\sigma}(q)$ while the third term represents the time taken to complete service of the rerouted users, that arrived at positions in between $\mathcal{C} \mid / \sigma$ and $\delta^{\sigma}(q)+|\mathcal{C}| / \sigma$. Note that $T_{n}^{\sigma}(q)=\phi_{n}^{\sigma}(0)-\phi_{n}^{\sigma}(q)$, hence is the time period between the time starts of gated service at external queues at stop 0 and the stop $\delta^{\sigma}(q)$ and so the time taken to service the rerouted users of the first stop, $\mathcal{T}_{r}([0,|\mathcal{C}| / \sigma])$, is not included in it. It instead includes the time taken to serve the rerouted users of the stop $\delta^{\sigma}(q), \mathcal{T}_{r}\left(\left[\delta^{\sigma}(q), \delta^{\sigma}(q)+|\mathcal{C}| / \sigma\right]\right)$. The + in the third term is in the modulo $|\mathcal{C}| / \sigma$ sense.

\subsection{First Moments}

We first obtain integral representation of the first moments of the workload, $\mathcal{T}_{e}\left([0, q], T_{n}^{\sigma}\right)$ using Lemma 1, [19] whose statement is reproduced here for easy reference.

Lemma 1. Let $T:[a, c] \mapsto \mathcal{R}^{+}$be either monotone (increasing or decreasing) or constant nonnegative random function on interval $[a, c]$. Then with $\tau(q):=E[T(q)]$ for all $q$,

$$
E\left[\mathcal{T}_{e}([a, c], T)\right]=\lambda \int_{a}^{c} b_{e}(q) f_{Q}(q) \tau(q) d q
$$

Similarly for rerouted users (proof in Appendix):

Lemma 2. With $T$ as in Lemma 1, the expected workload due to rerouted users equals (with $\tau(q):=E[T(q)]$ ):

$$
\begin{aligned}
E\left[\mathcal{T}_{r}([a, c], T)\right] & =\epsilon E\left[B_{r} 1_{\{R \in[a, c]\}}\right] \lambda \int_{0}^{|\mathcal{C}|} f_{Q}(q) \tau(q) d q \\
& =\epsilon \rho_{r}([a, c]) \int_{0}^{|\mathcal{C}|} f_{Q}(q) \tau(q) d q .
\end{aligned}
$$

Let $\tau_{n}^{\sigma}(q):=E\left[T_{n}^{\sigma}(q)\right]$ represent the first moment of $T_{n}^{\sigma}(q)$ when the number of stops equal $\sigma$ and let $\tau_{n}^{\infty}(q)$ represent the same for continuous system. Note that cycle time $C_{n}^{\sigma}(q)$ is the sum of monotone increasing $\left(q \mapsto T_{n}^{\sigma}(q)\right)$ and decreasing $\left(q \mapsto T_{n-1}^{\sigma}(|\mathcal{C}|)-T_{n-1}^{\sigma}(q)\right)$ random function of $q \in[0,|\mathcal{C}|]$. Further the workload that arrived in two non overlapping intervals is the sum of the workloads that arrived in individual intervals. Thus by Lemmas 1 and 2, by taking expectation over (8), we get:

$$
\begin{aligned}
\tau_{n}^{\sigma}(q) & =\delta^{\sigma}(q) \alpha^{-1}+\lambda \int_{0}^{\delta^{\sigma}(q)} b_{e}(y) c_{n-1}^{\sigma}(y) f_{Q}(y) d y \quad(10) \\
+ & \epsilon \rho_{r}\left(\left[\frac{|\mathcal{C}|}{\sigma}, \delta^{\sigma}(q)+\frac{|\mathcal{C}|}{\sigma}\right]\right) \int_{0}^{|\mathcal{C}|} c_{n-2}^{\sigma}(y) f_{Q}(y) d y \text { with } \\
c_{n}^{\sigma}(q) & =\tau_{n}^{\sigma}(q)+\tau_{n-1}^{\sigma}|\mathcal{C}|-\tau_{n-1}^{\sigma}(q) .
\end{aligned}
$$

Let $\mathcal{D}$ represent the Banach space of left continuous functions with right limits on $[0,|\mathcal{C}|]$ equipped with sup norm, $\|\tau\|_{\infty}:=\sup _{q \in[0,|\mathcal{C}|]}|\tau(q)|$. Let $\mathbb{N}:=\{1,2, \cdots, \infty\}$ be an Euclidean metric space. Define function $\mathcal{F}$, parametrized by $\sigma \in \mathbb{N}$, where the image $\mathcal{F}(\tau ; \sigma)$ is defined for any $\tau \in \mathcal{D}$, point-wise by:

$$
\begin{aligned}
& \mathcal{F}(\tau ; \sigma)(q):=\delta^{\sigma}(q) \alpha^{-1}+\tau(|\mathcal{C}|) \rho_{e}\left(\left[0, \delta^{\sigma}(q)\right]\right) \\
&+\tau(|\mathcal{C}|) \epsilon \rho_{r}\left(\left[\frac{|\mathcal{C}|}{\sigma}, \delta^{\sigma}(q)+\frac{|\mathcal{C}|}{\sigma}\right]\right) \text { for all } q \in[0,|\mathcal{C}|] .
\end{aligned}
$$

Let $\tau_{*}^{\sigma}(q), c_{*}^{\sigma}(q)$, for $\sigma \in \mathbb{N}$, represent stationary moments corresponding to $\tau_{n}^{\sigma}(q), c_{n}^{\sigma}(q)$ respectively. By stationarity ${ }^{2}$ and from equation (10), the stationary first moments of the discrete system is the fixed point of the parametrized function $\mathcal{F}$, at $\sigma<\infty$ while that of the continuous system is fixed point of the same function at $\sigma=\infty$.

The only limit point of the set $\mathbb{N}$ (in Euclidean metric) is $\infty$. Thus the function $\mathcal{F}$ is continuous in $(\tau, \sigma)$ (with $\tau \in \mathcal{D}$, $\sigma \in \mathbb{N}$ ) because: 1) $\mathcal{F}$ is bounded linear in $\tau$;2) as $\sigma \rightarrow \infty$, $\delta^{\sigma} \rightarrow \delta^{\infty}$ in sup norm and $\mathcal{F}$ is continuous in $\delta \in \mathcal{D}$ (when $\mathcal{F}$ is viewed as a function of $\tau, \delta, \sigma$ after replacing $\delta^{\sigma}$ with $\delta$ ). Hence (see proof of Theorem 2, [19] for more details) by contraction mapping theorem:

THEOREM 2. Assume $b_{e}, b_{r}, f_{Q}$ and $f_{R}$ are continuous in $q$. For any $\sigma$, the map $\mathcal{F}$ has an unique fixed point, $\tau_{*}^{\sigma}$,

\footnotetext{
${ }^{2}$ For any stationary point process, for example in our case $\left\{\phi_{n}^{\sigma}(q)\right\}$ for any fixed $q \in \mathcal{C}$ (for further explanations we consider $q=0$ and discuss the cycle times $\left.\left\{C_{n}^{\sigma}(0)\right\}_{n}\right)$, there will be two associated probabilities: Stationary and Palm Stationary $([1])$. In general, $\left\{\phi_{n}^{\sigma}(0)\right\}$ are defined such that $\phi_{0}^{\sigma}(0) \leq 0<\phi_{1}^{\sigma}(0)$. Palm probabilities are the stationary probabilities obtained after conditioning on the event that $\left\{\phi_{0}^{\sigma}(0)=0\right\}$ (see [1]). Throughout the paper, the expectation under Palm stationary measure is represented by $E^{0}$ and the corresponding moments are usually denoted with a * as under-script. In [1], the stationary moment of the residual cycle $C_{1}^{\sigma}(0)$ (which we refer as $C_{R}^{\sigma}$ ) as well as the past cycle $C_{0}^{\sigma}(0)$ (which we refer as $C_{P}^{\sigma}$ ) is obtained in terms of Palm probabilities as

$$
E\left[C_{R}^{\sigma}\right]=E\left[C_{P}^{\sigma}\right]=\frac{E^{0}\left[\left(C_{1}^{\sigma}\right)^{2}(0)\right]}{2 E^{0}\left[C_{1}^{\sigma}(0)\right]} .
$$

This result will be used in the next subsection. In Palm stationary regime, $\tau_{n}^{\sigma}, c_{n}^{\sigma}$ are same for all $n$, the common values are represented by $\tau_{*}^{\sigma}, c_{*}^{\sigma}$ and hence we get
} a fixed point operator, $\mathcal{F}$, to represent equation (10). 
if and only if $\rho=\rho_{e}+\epsilon \rho_{r}<1$. Further, $\tau_{*}^{\sigma}$, the stationary moments corresponding to discrete system with $\sigma$ stops, converges to that of the continuous system as $\sigma \rightarrow \infty$ :

$$
\sup _{q \in \mathcal{C}}\left|\tau_{*}^{\sigma}(q)-\tau_{*}^{\infty}(q)\right| \rightarrow 0 .
$$

Indeed because of modulo $|\mathcal{C}| / \sigma$ addition.

$$
\begin{aligned}
& \tau_{*}^{\sigma}(|\mathcal{C}|)=\frac{\delta^{\sigma}(|\mathcal{C}|) \alpha^{-1}}{1-\rho} \text { and } \\
& \tau_{*}^{\sigma}(q)=\delta^{\sigma}(q) \alpha^{-1}+\tau_{*}^{\sigma}(|\mathcal{C}|) \rho_{e}\left(\left[0, \delta^{\sigma}(q)\right]\right) \\
&+\epsilon \tau_{*}^{\sigma}(|\mathcal{C}|) \rho_{r}\left(\left[\frac{|\mathcal{C}|}{\sigma}, \delta^{\sigma}(q)+\frac{|\mathcal{C}|}{\sigma}\right]\right) \text { for all } q .
\end{aligned}
$$

\subsection{Second Moments}

The equivalent of Lemma 1 for the second moments is obtained in [19] (Lemma 2) and we reproduce it's statement:

Lemma 3. Assume the hypothesis of Lemma 1. Further, let $T_{1}$ be any positive random variable with $E\left[T_{1}\right]<\infty$. Then

$$
E\left[\mathcal{T}([a, c], T) T_{1}\right]=\lambda \int_{a}^{c} E\left[T(q) T_{1}\right] b_{e}(q) f_{Q}(q) d q .
$$

Using Lemma 3 and using the logic as in proof of Lemma 2 we can obtain the following lemma for the rerouted users:

Lemma 4. Assume the hypothesis of Lemma 3. Then, $E\left[\mathcal{T}_{r}([a, c], T) T_{1}\right]=\epsilon \rho_{r}([a, c]) \int_{0}^{|\mathcal{C}|} f_{Q}(q) E\left[T(q) T_{1}\right] d q$

We will be working with the following 3 second moments,

$$
\begin{aligned}
\tau_{n}^{(\sigma 2)}\left(q, q^{\prime}\right) & :=E\left[T_{n}^{\sigma}(q) T_{n}^{\sigma}\left(q^{\prime}\right)\right], \\
\tilde{\tau}_{n}^{(\sigma 2)}\left(q, q^{\prime}\right) & :=E\left[T_{n}^{\sigma}(q) T_{n-1}^{\sigma}\left(q^{\prime}\right)\right] \text { and } \\
\ddot{\tau}_{n}^{(\sigma 2)}\left(q, q^{\prime}\right) & :=E\left[T_{n}^{\sigma}(q) T_{n-2}^{\sigma}\left(q^{\prime}\right)\right] .
\end{aligned}
$$

The fixed point equations for these moments will be shown to be equal to the sum of the operators, $\Omega+\Upsilon$ and these operators are all given at the top of the next page. We begin with $\tau^{(2)}$. Using Lemmas 3, 4 and by taking expectation after multiplying equation (8) with $T_{n}^{\sigma}\left(q^{\prime}\right)$ we get:

$$
\begin{aligned}
\tau_{n}^{(\sigma 2)}\left(q, q^{\prime}\right) & \\
= & \delta^{\sigma}(q) \alpha^{-1} \tau_{n}^{\sigma}\left(q^{\prime}\right)+E\left[\mathcal{T}_{e}\left(\left[0, \delta^{\sigma}(q)\right], C_{n-1}^{\sigma}\right) T_{n}^{\sigma}\left(q^{\prime}\right)\right] \\
& +E\left[\mathcal{T}_{r}\left(\left[\frac{|\mathcal{C}|}{\sigma}, \delta^{\sigma}(q)+\frac{|\mathcal{C}|}{\sigma}\right], C_{n-2}^{\sigma}\right) T_{n}^{\sigma}\left(q^{\prime}\right)\right] \\
& =\Upsilon_{q, q^{\prime}}\left(\tau_{n}^{\sigma} ; \sigma\right)+\Omega_{q, q^{\prime}}\left(\tau_{n}^{(2)}, \tilde{\tau}_{n}^{(\sigma 2)}, \ddot{\tau}_{n}^{(\sigma 2)} ; \sigma\right) .
\end{aligned}
$$

Similarly, multiplying (8) respectively with $T_{n-1}^{\sigma}\left(q^{\prime}\right)$ and $T_{n-2}^{\sigma}\left(q^{\prime}\right)$ and then taking the expectation we obtain:

$\tilde{\tau}_{n}^{(\sigma 2)}\left(q, q^{\prime}\right)=\Upsilon_{q, q^{\prime}}\left(\tau_{n-1}^{\sigma} ; \sigma\right)+\tilde{\Omega}_{q, q^{\prime}}\left(\tau_{n-1}^{(\sigma 2)}, \tilde{\tau}_{n}^{(\sigma 2)}, \tilde{\tau}_{n-1}^{(\sigma 2)} ; \sigma\right)$, $\ddot{\tau}_{n}^{(\sigma 2)}\left(q, q^{\prime}\right)=\Upsilon_{q, q^{\prime}}\left(\tau_{n-2}^{\sigma} ; \sigma\right)+\ddot{\Omega}_{q, q^{\prime}}\left(\tau_{n-2}^{(\sigma 2)}, \tilde{\tau}_{n-1}^{(\sigma 2)}, \ddot{\tau}_{n}^{(\sigma 2)} ; \sigma\right)$.

Let $\tau_{*}^{(\sigma 2)}, \tilde{\tau}_{*}^{(\sigma 2)}$ and $\ddot{\tau}_{*}^{(\sigma 2)}$ represent respectively the stationary moments corresponding to $\tau_{n}^{(\sigma 2)}, \tilde{\tau}_{n}^{(\sigma 2)}$ and $\ddot{\tau}_{n}^{(\sigma 2)}$. Let $\top$ represent the ordered triplet $\left(\tau^{(2)}, \tilde{\tau}^{(2)}, \ddot{\tau}^{(2)}\right)$ and define

$$
\begin{aligned}
& \Theta_{q, q^{\prime}}^{\tau}(\top ; \sigma)=\Upsilon_{q, q^{\prime}}\left(\tau_{*}^{\sigma}\right)+\Omega_{q, q^{\prime}}(\top ; \sigma) \\
& \Theta_{q, q^{\prime}}^{\tilde{\tau}}(\top ; \sigma)=\Upsilon_{q, q^{\prime}}\left(\tau_{*}^{\sigma}\right)+\tilde{\Omega}_{q, q^{\prime}}\left(\tau^{(2)}, \tilde{\tau}^{(2)}, \tilde{\tau}^{(2)} ; \sigma\right),(16) \\
& \Theta_{q, q^{\prime}}^{\ddot{\tau}}(\top ; \sigma)=\Upsilon_{q, q^{\prime}}\left(\tau_{*}^{\sigma}\right)+\ddot{\Omega}_{q, q^{\prime}}(\top ; \sigma)
\end{aligned}
$$

where $\tau_{*}^{\sigma}$ are given by Theorem 2 (note these are continuous as $\sigma \rightarrow \infty)$. The (Palm) stationary second moments, $\top_{*}^{\sigma}$, of the discrete system are the fixed points of the map, $\Theta:=$ $\left(\Theta^{\tau}, \Theta^{\tilde{\tau}}, \Theta^{\ddot{\tau}}\right)$, at $\sigma<\infty$, while that of the continuous system are fixed points of the same map when $\sigma=\infty$. We show that these moments of the discrete system converge to that of the continuous system (proof in Appendix):

TheOREM 3. Assume $b_{e}, b_{r}, f_{Q}$ and $f_{R}$ are continuous. Then there exists a threshold $\lambda_{0}$ (given by equation (26) in the proof) and for all Poisson arrival rates less than $\lambda \leq \lambda_{0}$, the stationary second moments $\top_{*}^{\sigma}$ converge uniformly in $q$ :

$$
\sup _{q, q^{\prime} \in[0,|C|]}\left|\top_{*}^{\sigma}\left(q, q^{\prime}\right)-\top_{*}^{\infty}\left(q, q^{\prime}\right)\right| \rightarrow 0 \text { as } \sigma \rightarrow \infty \text {. }
$$

\section{PROOF OF THEOREM 1}

By Theorems 2, 3 the first and second order stationary moments of $T_{n}$, of the discretized polling system, converge towards the corresponding ones of the continuous polling system, in supremum norm, i.e., uniformly of the positions $q, q^{\prime}$ on the circle. We obtain the expression for the stationary expected virtual workload using these moments for both the discrete and continuous polling system and then complete the proof of the Theorem 1.

The first two palm stationary moments of the cycle time, $C_{n}^{\sigma}(q)$ defined in (7), $E^{0}\left[C_{n}^{\sigma}(q)\right]$ and $E^{0}\left[\left(C_{n}^{\sigma}(q)\right)^{2}\right]$ equal:

$$
\begin{aligned}
c_{*}^{\sigma}(q)= & \tau_{*}^{\sigma}(|\mathcal{C}|), \\
c_{*}^{(\sigma 2)}(q, q)= & 2 \tau_{*}^{(\sigma 2)}(q, q)+\tau_{*}^{(\sigma 2)}(|\mathcal{C}|,|\mathcal{C}|)+2 \tilde{\tau}_{*}^{(\sigma 2)}(q,|\mathcal{C}|) \\
& -2 \tilde{\tau}_{*}^{(\sigma 2)}(q, q)-2 \tau_{*}^{(\sigma 2)}(q,|\mathcal{C}|)
\end{aligned}
$$

Thus the stationary first moment of the residual of the cycle $C_{n}^{\sigma}(q)$ as seen by a random user equals (see footnote 2 ):

$$
E\left[C_{R}^{\sigma}(q)\right]=\frac{c_{*}^{(\sigma 2)}(q, q)}{2\left(c_{*}^{\sigma}(q)\right)^{2}} .
$$

In the following we calculate the stationary expected workload due to external users and rerouted users separately, in terms of the stationary moments of the previous section, using Little's law and then the Wald's Lemma.

Workload due to External users: A randomly arriving external user, arriving at $q$, has to wait for:

1) residual of his own cycle $C(q), E\left[C_{R}^{\sigma}(q)\right]$;

2) in a discrete system (because of arrival position order service), till the external users placed geographically above him are served, which by Lemma 1 equals

$$
E\left[\mathcal{T}_{e}\left(\left[\delta^{\sigma}(q), q\right], C\right)\right]=\tau_{*}^{\sigma}(|\mathcal{C}|) \rho_{e}\left(\left[\delta^{\sigma}(q), q\right]\right) .
$$

Therefore the expected waiting time of a external user is:

$E\left[W_{e}^{\sigma}\right](q)=E\left[C_{R}^{\sigma}(q)\right]+1_{\{\sigma<\infty\}} \tau_{*}^{\sigma}(|\mathcal{C}|) \rho_{e}\left(\left[\delta^{\sigma}(q), q\right]\right)$. 


$$
\begin{aligned}
\Omega_{q, q^{\prime}}\left(\tau^{(2)}, \tilde{\tau}^{(2)}, \ddot{\tau}^{(2)} ; \sigma\right):= & \lambda \int_{0}^{\delta^{\sigma}(q)} b_{e}(y) f_{Q}(y)\left(\tau^{(2)}\left(q^{\prime}, y\right)+\tilde{\tau}^{(2)}\left(q^{\prime},|\mathcal{C}|\right)-\tilde{\tau}^{(2)}\left(q^{\prime}, y\right)\right) d y \\
& +\epsilon \rho_{r}\left(\left[\frac{|\mathcal{C}|}{\sigma}, \delta^{\sigma}(q)+\frac{|\mathcal{C}|}{\sigma}\right]\right) \int_{0}^{|\mathcal{C}|} f_{Q}(y)\left(\tilde{\tau}^{(2)}\left(q^{\prime}, y\right)+\ddot{\tau}^{(2)}\left(q^{\prime},|\mathcal{C}|\right)-\ddot{\tau}^{(2)}\left(q^{\prime}, y\right)\right) d y \\
\tilde{\Omega}_{q, q^{\prime}}\left(\tau^{(2)}, \tilde{\tau}_{+}^{(2)}, \tilde{\tau}_{-}^{(2)} ; \sigma\right):= & \lambda \int_{0}^{\delta^{\sigma}(q)} b_{e}(y) f_{Q}(y)\left(\tilde{\tau}_{+}^{(2)}\left(y, q^{\prime}\right)+\tau^{(2)}\left(|\mathcal{C}|, q^{\prime}\right)-\tau^{(2)}\left(y, q^{\prime}\right)\right) d y \\
& +\epsilon \rho_{r}\left(\left[\frac{|\mathcal{C}|}{\sigma}, \delta^{\sigma}(q)+\frac{|\mathcal{C}|}{\sigma}\right]\right) \int_{0}^{|\mathcal{C}|} f_{Q}(y)\left(\tau^{(2)}\left(q^{\prime}, y\right)+\tilde{\tau}_{-}^{(2)}\left(q^{\prime},|\mathcal{C}|\right)-\tilde{\tau}_{-}^{(2)}\left(q^{\prime}, y\right)\right) d y \\
\ddot{\Omega}_{q, q^{\prime}}\left(\tau^{(2)}, \tilde{\tau}^{(2)}, \ddot{\tau}^{(2)} ; \sigma\right):= & \lambda \int_{0}^{\delta^{\sigma}(q)} b_{e}(y) f_{Q}(y)\left(\ddot{\tau}^{(2)}\left(y, q^{\prime}\right)+\tilde{\tau}^{(2)}\left(|\mathcal{C}|, q^{\prime}\right)-\tilde{\tau}^{(2)}\left(y, q^{\prime}\right)\right) d y \\
& +\epsilon \rho_{r}\left(\left[\frac{|\mathcal{C}|}{\sigma}, \delta^{\sigma}(q)+\frac{|\mathcal{C}|}{\sigma}\right]\right) \int_{0}^{|\mathcal{C}|} f_{Q}(y)\left(\tilde{\tau}^{(2)}\left(y, q^{\prime}\right)+\tau^{(2)}\left(|\mathcal{C}|, q^{\prime}\right)-\tau^{(2)}\left(y, q^{\prime}\right)\right) d y \\
\Upsilon_{q, q^{\prime}}(\tau ; \sigma):= & \delta^{\sigma}(q) \alpha^{-1} \tau\left(q^{\prime}\right)
\end{aligned}
$$

\section{The Fixed point equations for the second moments}

By Little's law ([24]), the density of the stationary number of waiting users (awaiting first service) is $\lambda f_{Q}(q) E\left[W_{e}^{\sigma}\right](q)$. By Wald's lemma the density of the expected stationary workload corresponding to external users is $\lambda f_{Q}(q) E\left[W_{e}^{\sigma}\right](q) b_{e}(q)$. Thus the expected stationary workload due to all the external users (awaiting the first service) is:

$$
\int_{0}^{|\mathcal{C}|} \lambda f_{Q}(q) E_{e}^{\sigma}[W](q) b_{e}(q) d q
$$

Workload due to Rerouted users: A rerouted user arrives just after completion of his first service and because of immediate rerouting to another point in the circle. Consider one such user who arrived at a point $q$ in $\mathcal{C}$. His waiting time depends upon the point at which his first service was completed. Conditioned that the position of the first service was at $q^{\prime}$ (whose distribution is given by $P_{Q}\left(d q^{\prime}\right)=f_{Q}\left(q^{\prime}\right) d q^{\prime}$ because of independence), he will have to wait on average for: 1) if $q^{\prime}<q$ then $\tau_{*}^{\sigma}(q)-\tau_{*}^{\sigma}\left(q^{\prime}\right)$ period of time; 2) if $q^{\prime}>q$ then $\tau_{*}^{\sigma}(|\mathcal{C}|)-\tau_{*}^{\sigma}\left(q^{\prime}\right)+\tau_{*}^{\sigma}(q)$ period of time. The above gives the waiting time till the point at which the external arrivals belong to his strip $\left[\delta^{\sigma}(q), \delta^{\sigma}(q)+|\mathcal{C}| / \sigma\right)$ are served. But (in case of discrete systems) the rerouted user will be served before this time point and the exact difference in the two time stamps would be the time taken to serve the rerouted users belonging to strip $\left[q, \delta^{\sigma}(q)+|\mathcal{C}| / \sigma\right)$ (because of arrival position order service). Thus the stationary average waiting time of a rerouted user arrived at point $q$, using Lemma 2 :

$$
\begin{aligned}
& E\left[W_{r}^{\sigma}\right](q)=\int_{q}^{|\mathcal{C}|}\left(\tau_{*}^{\sigma}(|\mathcal{C}|)-\tau_{*}^{\sigma}\left(q^{\prime}\right)+\tau_{*}^{\sigma}(q)\right) f_{Q}\left(q^{\prime}\right) d q^{\prime} \\
& +\int_{0}^{q}\left(\tau_{*}^{\sigma}(q)-\tau_{*}^{\sigma}(y)\right) f_{Q}(y) d y-E\left[\mathcal{T}_{r}\left(\left[q, \delta^{\sigma}(q)+\frac{|\mathcal{C}|}{\sigma}\right], C\right)\right] \\
& =\tau_{*}^{\sigma}(q)+\tau_{*}^{\sigma}(|\mathcal{C}|) P_{Q}([q,|\mathcal{C}|])-\int_{0}^{|\mathcal{C}|} \tau_{*}^{\sigma}\left(q^{\prime}\right) f_{Q}\left(q^{\prime}\right) d q^{\prime} \\
& -\epsilon \rho_{r}\left(\left[q, \delta^{\sigma}(q)+\frac{|\mathcal{C}|}{\sigma}\right]\right) \tau_{*}^{\sigma}(|\mathcal{C}|) .
\end{aligned}
$$

By Little's law ([24]) the density of the stationary number of waiting rerouted users is $\lambda \epsilon f_{R}(q) E\left[W_{r}^{\sigma}(q)\right]$ and by Wald's lemma the density of the expected stationary workload corresponding to rerouted users is $\lambda \epsilon f_{R}(q) E\left[W_{r}^{\sigma}\right](q) b_{r}(q)$.

Total expected stationary workload: Therefore, the total expected stationary virtual workload is given by equation (22) placed at the top of the next page. In the above $W^{\infty}$ represents the stationary expected virtual workload for the continuous system. By the Theorems 2, 3 the terms inside the first integral of (22) (see also (18) and (19)), defining the expected virtual workload, converge towards that of the continuous polling system point-wise and uniformly in $q$. And the terms in the second integral converge to zero point-wise as $\sigma \rightarrow \infty$ and the convergence can once again be shown to be uniform, for example (for some $\nu<\infty$ )

$$
\rho_{e}\left(\left[\delta^{\sigma}(q), q\right]\right) \leq \lambda\left\|b_{e}\right\|_{\infty}\left\|f_{Q}\right\|_{\infty}\left|q-\delta^{\sigma}(q)\right| \leq \frac{\nu}{\sigma} \text { for all } q .
$$

So by bounded convergence theorem, the expected stationary virtual workload of the discrete polling system converges towards that of the continuous polling system as $\sigma \rightarrow \infty$.

The expected virtual workload for each $\sigma$ is obtained in section 3 using results of [15] as equation (5) and it's limit (1) represents the stationary expected workload of the continuous system. This completes the proof of Theorem 1.

The stationary expected workload formula of Theorem 1 can be used for obtaining the performance of many systems. We used the formula (1) for obtaining the performance of a ferry based wireless LANs (FWLAN) in the following.

\section{FERRY BASED WIRELESS LAN}

Static users are scattered in a geographical area $\Delta$. The network is sparse and there is no direct global connectivity. The actual communication is facilitated by a ferry which moves in a closed cyclic path $\mathcal{C}$, placed inside $\Delta$, repeatedly with constant speed $\alpha$ and serves as a postman. Every time the ferry stops, it spends extra time $t_{a}$ for accelerating. The ferry collects the data from the source users as and when it encounters one. We call this as uplink service. The uplink 


$$
\begin{gathered}
\mathcal{W}^{\sigma}=\lambda \int_{0}^{|\mathcal{C}|}\left[E\left[C_{R}^{\sigma}(q)\right] b_{e}(q) f_{Q}(q)+\left(\tau_{*}^{\sigma}(q)+\tau_{*}^{\sigma}(|\mathcal{C}|) P_{Q}([q,|\mathcal{C}|])-\int_{0}^{|\mathcal{C}|} \tau_{*}^{\sigma}\left(q^{\prime}\right) f_{Q}\left(q^{\prime}\right) d q^{\prime}\right) b_{r}(q) \epsilon f_{R}(q)\right] d q \\
+1_{\{\sigma<\infty\}} \lambda \int_{0}^{|\mathcal{C}|}\left[\tau_{*}^{\sigma}(|\mathcal{C}|) \rho_{e}\left(\left[\delta^{\sigma}(q), q\right]\right) b_{e}(q) f_{Q}(q)-\epsilon \rho_{r}\left(\left[q, \delta^{\sigma}(q)+\frac{|\mathcal{C}|}{\sigma}\right]\right) \tau_{*}^{\sigma}(|\mathcal{C}|) b_{r}(q) \epsilon f_{R}(q)\right] d q
\end{gathered}
$$

data also comes with the address of the destination user to which it is intended. The ferry downloads the data to the destined user the first time it meets the later, after collecting the uplink data. We refer this service as downlink service.

The base station (BS) forms a global gateway to the external world. The communication between the users of the network and the BS is also established via ferry as is done between two users of the node. Every download starts with uplink from BS to the ferry followed by the downlink from the ferry to the destined user and every upload starts with uplink from the source user to the ferry followed by the downlink from ferry to the BS. Thus the BS is like any other user.

Each point $q$ in the cyclic path is assigned with a set of points $I(q) \subset \Delta$ and ferry stops at $q$ if there is an user in $I(q)$ with either downlink or uplink request. For example, if we consider $\Delta$ to be an annular ring, the ferry is moving along a co-centric circle and if the sets $\{I(q)\}$ are based on the nearest point criterion then the Ferry will stop at a point on the circle if there is an user located at the same angle (see Figure 1).

The data transfer request arrivals are modeled as Poisson arrivals and each of these arrivals are associated with two marks: $X$ the position of source distributed as $P_{X}$ and $Y$ the position of the destination/sink distributed as $P_{Y}$. Every such request requires fixed size, $\eta$, of data to be transfered from source $X$ to destination $Y$. The position of the source and destination are independent of each other.

Ferry uses a wireless link to serve the users. It can receive/transmit the messages from/to the users, at a distance of $d$ from it, at a rate $\kappa(d)$ for some decreasing function $\kappa$. Thus the total time required for transmitting a message of size $\eta$, when the user is located at $x \in \Delta$ and is associated with $q(x) \in \mathcal{C}$ is equal to its size divided by the service rate:

$$
B(X)=\frac{\eta}{\kappa(\|q(x)-x\|)} .
$$

\subsection{Mapping to a Continuous Polling system}

We analyze this FWLAN using Theorem 1. We begin with identifying the components of the continuous polling system.

Server and path of the polling system : The ferry represents the server of the polling system. The ferry stops at a point $q$ in its path only when there is a user with (downlink/uplink) request anywhere on the strip $I(q)$. Thus the entire strip $I(q)$ is modeled as a point on the server's path, in an equivalent continuous polling system.

Service times: The time $t_{a}$ for acceleration is required only when the ferry stops and hence is added to the equivalent service time. An arrival $(X, Y)$ is associated with the points
$q(X), q(Y)$ of the ferry route if $X, Y$ lie in corresponding strips, i.e., if $X \in I(q(X))$ and $Y \in I(q(Y))$. Thus time required for uplink and downlink services respectively are:

$$
B(X)=\frac{\eta}{\kappa(|X-q(X)|)}+t_{a} \text { and } B(Y)=\frac{\eta}{\kappa(|Y-q(Y)|)}+t_{a}
$$

The moments of the uplink or downlink service times, in general depend upon the point $q \in \mathcal{C}$. For uplink,

$$
\begin{aligned}
b_{u}(q) & =E[B(X) \mid q(X)=q] \\
& =E_{X}\left[\frac{\eta}{\kappa(|q-X|)} \mid X \in I(q)\right]+t_{a} \text { and } \\
b_{u}^{(2)}(q) & =E_{X}\left[\frac{\eta^{2}}{\kappa(|q-X|)^{2}} \mid X \in I(q)\right]-t_{a}^{2}+2 t_{a} b_{u}(q) .
\end{aligned}
$$

Downlink moments $b_{d}, b_{d}^{(q)}$ can be defined in a similar way. Note that $b_{u}, b_{u}^{(2)}, b_{d}$ and $b_{d}^{(2)}$ respectively represent the quantities $b_{e}, b_{e}^{(2)}, b_{r}$ and $b_{r}^{(2)}$ of Theorem 1 .

External and Rerouted arrivals: Every data transfer requires two services: starts with uplink service and ends with downlink service. A Poisson process models the arrival of a data transfer request and the same marks the arrival of an uplink service requirement. Hence, uplinks represent the external arrivals to the polling system that models the FWLAN. The completion of uplink service marks the arrival of a downlink service and hence the downlinks represent the rerouted arrivals. Every uplink leads to a downlink and hence $\epsilon=1$.

Position of arrival in the 'polling system' : The position of uplink arrival in FWLAN is given by $P_{X}$, a distribution over $\Delta$. Every arrival in the strip $I(q)$ marks the arrival at point $q$ of $\mathcal{C}$ in the equivalent polling system. Thus,

$$
P_{Q}(A):=P_{X}\left(\cup_{q \in A} I(q)\right) \text { for any Borel set } A \subset \mathcal{C},
$$

represents the external arrival distribution. For example, in an annular ring with circular path, i.e., for some $h_{1} \leq l \leq h_{2}$

$$
\Delta:=\left\{x \in \mathcal{R}^{2}: h_{1}^{2} \leq|x| \leq h_{2}^{2}\right\} \text { and } \mathcal{C}:=\{q:\|q\|=l\},
$$

and if $P_{X} \sim \mathcal{U}(\Delta)$ (uniform distribution) and $I(q)=\{x$ : $\angle x=\angle q\}$ (with $\angle x$ representing the angle made by the line joining $0, x$ with the $x$ axis), then $P_{Q}$ will be uniform over $\mathcal{C}$. Similarly $P_{R}(A):=P_{Y}\left(\cup_{q \in A} I(q)\right)$, for all Borel $A \subset \mathcal{C}$.

Thus the FWLAN can be modeled by a continuous polling system with rerouting, Theorem 1 can be applied and the stationary expected workload of the FWLAN can be calculated using (1) for any given cyclic path $\mathcal{C}$ and the corresponding line segments $\left\{I_{q}\right\}_{q \in \mathcal{C}}$.

In the following we consider a simple example of FWLAN (i.e., $\mathcal{C}$ and line segments $\left\{I_{q}\right\}$ ) and apply Theorem 1. In [20] we obtained an extension of Theorem 1, which helped in modeling many more practical examples of FWLAN. In 
particular we consider a ferry moving in a rectangular path, like that of a route of a bus, in [20]. The results of this paper can model simple examples and pave way for more complicated analysis, as done in [20], which then can model really interesting realistic examples.

\subsection{Optimal Ferry path}

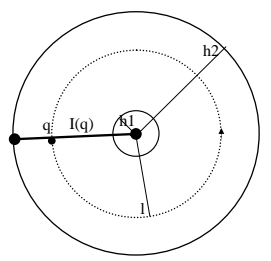

Figure 1: Ferry in an annular ring

We consider a simple setting and design an optimal route for the ferry among a given set of possible routes. We consider the following configuration of FWLAN: 1) annular ring $\left.\Delta:=\left\{x \in \mathcal{R}^{2}: h_{1}^{2} \leq|x| \leq h_{2}^{2}\right\} ; 2\right)$ uniform arrivals i.e., $P_{X} \sim \mathcal{U}(\Delta)$ and so is $\left.P_{Y} ; 3\right)$ rate function resulting from the losses in wireless medium considers only the direct path for attenuation and is calculated assuming a receive transmit antennae difference of 1 and pathloss factor $\beta$ :

$$
\kappa(d)=\left(1+d^{2}\right)^{-\beta / 2} .
$$

The ferry moves on one concentric circle of radius $l$ in the annular ring (Figure 1), i.e., $\mathcal{C}_{l}=\left\{q \in \mathcal{R}^{2}:\|q\|=l\right\}$. The best possible design with $\kappa$ as above, is to associate every arrival with the nearest point on the ferry route, i.e., $X$ is associated with $q(X):=\arg \min _{q \in \mathcal{C}_{l}}|q-X|$. Hence, $I(q)=\{x \in \Delta: \angle(x)=\angle(q)\}$, the angular segments for all $q \in \mathcal{C}_{l}$, (see figure 1). Aim is to find the optimal radius:

$$
l^{*}=\arg \min _{l \in\left[h_{1}, h_{2}\right]} V_{\text {fwlan }}\left(l ; h_{1}, h_{2}\right)
$$

where $V_{\text {fwlan }}\left(l ; h_{1}, h_{2}\right)$ represents the expected stationary workload in FWLAN when the ferry moves in $\mathcal{C}_{l}$.

Since $\{I(q)\}$ are angular segments, for calculating the service time moments one will require the radius $\Gamma=\|X\|$ of the arrival. Under the assumptions of this section, $\Gamma \sim$ $2 r d r /\left(h_{2}^{2}-h_{1}^{2}\right)$ and further because of symmetry the service time moments will be independent of the position $q \in \mathcal{C}_{l}$, are same for uplink and downlink, but depend upon the ferry path radius $l$. The common service moments are:

$$
\begin{aligned}
\bar{b} & =\eta \int_{h_{1}}^{h_{2}}\left(1+(r-l)^{2}\right)^{\beta / 2} \frac{2 r d r}{h_{2}^{2}-h_{1}^{2}}+t_{a} \text { and } \\
\bar{b}^{(2)} & =\eta^{2} \int_{h_{1}}^{h_{2}}\left(1+(r-l)^{2}\right)^{\beta} \frac{2 r d r}{h_{2}^{2}-h_{1}^{2}}-t_{a}^{2}+2 t_{a} \bar{b} .
\end{aligned}
$$

The stationary expected workload (1) for this symmetric settings is simplified as (2). Using this, $\rho=2 \lambda \bar{b}$ and

$$
V_{\text {fwlan }}^{\text {sym }}\left(l ; h_{1}, h_{2}\right)=\frac{\rho \lambda\left(\bar{b}^{(2)}+\bar{b}^{2}\right)}{1-\rho}+\frac{3 \pi l \rho \alpha^{-1}}{2(1-\rho)} .
$$

One has to perform numerical computations using the above formula to obtain the optimal radius. This could be done even for more complicated settings of FWLAN. However one can get the following asymptotic characteristic of $l^{*}$ from the formula itself: 1) as the propagation coefficient $\beta$ tends to zero the moments $\bar{b}, \bar{b}^{(2)}$ become independent of $l$ and hence $l^{*}$ tends to $h_{1}$, i.e., the optimal path for the ferry is the inner circle. (2) as the speed of the ferry, $\alpha$, increases to infinity, the second term of (24) becomes negligible and then the optimal radius is influenced mainly by the service time moments and so $l^{*}$ approximately optimizes $\bar{b}$ and hence is above the middle of the ring, i.e., larger than $\left(h_{1}+h_{2}\right) / 2$.

\section{CONCLUSIONS}

We study a continuous polling system in which the users can be rerouted to a new position with a second service request after completion of their first service. We obtain an expression for the expected stationary workload. We obtain this result under more general conditions than the usual symmetric conditions. We use the approach of discretization so that the available Pseudo conservation laws of discrete polling systems with rerouting ([15]) can be utilized. We expressed the expected workload as a parametrized function of moment fixed points. The later are some stationary moments obtained as fixed points of a function defined on spaces of left continuous functions with right limits equipped with supremum norm and which are further parametrized by the number of discretization levels. We show the required convergence via the continuous dependence of the fixed points on the parameter. This way we obtained a common expression, which represents the expected virtual workload for continuous as well as the discretized polling systems, at different values of the parameter. We then showed the continuity of the expected virtual workload with respect to the parameter and hence obtained the expected virtual workload for the continuous system as the limit of the expected virtual workloads of the discrete systems, when the levels of discretization tend to infinity. We applied these results to a wireless LAN in which a ferry assists data transfer among the users of the network as well as the users and the gateway to the external world.

This is a second paper using this approach and we earlier obtained using similar approach the performance of a continuous polling system that supports mixed service disciplines. In ([20]), we further generalized Theorem 1, to support any finite number of reroutings. Further, the distribution of the position of arrivals can be a mixture of discrete and continuous probabilities.

\section{Appendix}

Proof of Lemma 2 : Let $N$ represent the number of users that caused the workload $\mathcal{T}_{e}([0,|\mathcal{C}|], T)$. Note that $N=$ $\hat{\mathcal{T}}_{e}([0,|\mathcal{C}|], T)$, where $\hat{\mathcal{T}}_{e}([0,|\mathcal{C}|], T)$ represents the workload due to the arrivals which carry in unit service time (i.e., $\hat{\mathcal{T}}$ is with $B_{e} \equiv 1$, so $b_{e}(q)=1$ for all $\left.q\right)$. Hence by Lemma 1

$$
E[N]=\lambda \int_{0}^{|\mathcal{C}|} f_{Q}(q) \tau(q) d q \text { where } \tau(q)=E[T(q)] .
$$

Let $N_{r}$ represent the number of rerouted users among these $N$. Then

$$
N_{r}=\sum_{i=1}^{N} 1_{\{\text {user } i \text { rerouted to }[a, c]\}} .
$$

The user after first service reroutes himself independent of everything else and hence by applying Wald's lemma to (25):

$$
E\left[N_{r}\right]=E[N] E\left[1_{\{\text {Reroute to }[a, c]\}}\right]=E[N] \epsilon P_{R}([a, c]) .
$$


The service time requirements in the second service $B_{r}$ is independent of every other process and its average conditioned that the arrival is in interval $[a, c]$ is $E\left[B_{r} \mid R \in[a, c]\right]$ and hence by applying Wald's lemma again:

$$
E\left[\mathcal{T}_{r}([a, c], T)\right]=\epsilon E[N] E\left[B_{r} 1_{\{R \in[a, c]\}}\right] .
$$

Proof of Theorem 3: The required stationary second moments are the fixed point of the function $\Theta:=\left(\Theta^{\tau}, \Theta^{\tilde{\tau}}, \Theta^{\ddot{\tau}}\right)$ defined in equations (15)-(17), parametrized by $\sigma$. We prove the theorem in the following steps.

Step 1: Spaces: We consider function $\Theta$ defined over the spaces: 1) $\top$ over the Banach space $\left(\mathcal{D}^{(2)}\right)^{3}$, where $\mathcal{D}^{(2)}:=$ $\mathcal{D}([0,|\mathcal{C}|] \times[0,|\mathcal{C}|])$, is the space of left continuous functions with right limits on $[0,|\mathcal{C}|] \times[0,|\mathcal{C}|]$ equipped with sup norm; 2) $\sigma$ is in Metric space $\mathbb{N}$ equipped with Euclidean metric.

Step 2: Smoothness properties of the map $\Theta$ : It is easy to see by boundedness that $\Theta$ is continuous and linear in variable $\top:=\left(\tau^{(2)}, \tilde{\tau}^{(2)}, \ddot{\tau}^{(2)}\right)$. The continuity of $\Omega$ functions (defined in (11)-(13)) with respect to $\sigma$ (the only limit point is at $\infty$, i.e., as $\sigma \rightarrow \infty)$ is readily seen by inspection itself while the continuity of the function

$$
\sigma \mapsto \Upsilon\left(\tau_{\delta}^{*}\right) \text { as } \sigma \rightarrow \infty
$$

in sup norm is given by Theorem 2. Thus the function $\Theta$ defined in (15)-(17) is continuous at $\sigma=\infty$.

Step 3: Contraction: From equations (15)-(17), for any $\sigma$

$$
\begin{gathered}
\left\|\Omega\left(\top_{1} ; \sigma\right)-\Omega\left(\top_{2} ; \sigma\right)\right\|_{\infty} \leq 3 \rho\left\|\top_{1}-\top_{2}\right\|_{\infty} . \\
\text { Let } \lambda_{0}:=\frac{1}{3\left(\int_{0}^{|\mathcal{C}|} b_{e}(q) f_{Q}(q) d q+\epsilon \int_{0}^{|\mathcal{C}|} b_{r}(q) f_{R}(q) d q\right)} .
\end{gathered}
$$

For all $\lambda<\lambda_{0}, 3 \rho<1$ and so $\Theta$ is a contraction for all $\sigma$.

Step 4: The Result : By the Contraction Mapping Theorem (Corollary 3.1.4, page 112, [2]) we obtain: 1) the existence of unique fixed point for all $\sigma ; 2$ ) the continuous dependence upon the parameter, $\sigma$. Thus, the fixed points of $\Omega$ with $\sigma<\infty$, i.e., the stationary second moments of the discrete system converge to the corresponding values in continuous system (with $\sigma=\infty$ ) as $\sigma \rightarrow \infty$, in sup norm.

\section{REFERENCES}

[1] F. Baccelli and P. Brémaud. Elements of Queueing Theory. volume 26. Applications of Mathematics, Springer-Verlag, 1991.

[2] M. S. Berger. Nonlinearity and Functional Analysis. Academic Press, New York, 1977.

[3] J. E.G. Coffman and E. Gilbert. A continuous polling system with constant service times. IEEE Trans. on Information Theory, 584-591, 1986.

[4] J. E.G. Coffman and E. Gilbert. Polling and greedy servers on the line. Queueing Systems 2115-145, 1987.

[5] I. Eliazar. The snowblower problem. Queueing Systems, 45, 357-380, 2003.

[6] I. Eliazar. From polling to snowplowing. Queueing Systems, 51(1-2), 115-133, 2005.
[7] S. Fuhrmann and R. Copper. Applications of the decomposition principle in $\mathrm{M} / \mathrm{G} / 1$ vacation models to two continuum cyclic queueing models. ATEST Tech. J. 64 1091-1098, 1985.

[8] D. Kroese and V. Schmidt. Queueing systems on the circle. Z. Oper. Res. 37(3) 303-331, 1993.

[9] D. Kroese and V. Schmidt. Single-server queues with spatially distributed arrivals. Queueing Systems, 17, 317-345, 1994.

[10] D. Kroese and V. Schmidt. Light-traffic analysis for queues with spatially distributed arrivals. Math. Oper. Research, 21, 135-15\%, 1996.

[11] D. Kroese and V. Schmidt. A continuous polling system with general service times. The Annals of Applied Probability, Vol. 2, No. 4, 906-927, Nov 1992.

[12] E. Altman and H. Levy. Queueing in space. Advances of Applied Probability, vol. 11, no. 1-2, 35-57, 1994.

[13] E. Altman, P. Konstantopoulos and Z. liu. Stability, monotonicity and invariant quantities in general polling systems. Queuing Syst., vol. 11, no. 1-2, 35-57, 1992.

[14] L. Georgiadis and W. Szpankowski. Stability of token passing ring. Queuing Syst., vol. 11, no. 1-2, 7-33, 1992.

[15] M. Sidi, H. Levy and S. W. Fuhrmann. A queuing network with a single cyclically roving server. Queuing Systems, 11, (special issue on Polling Models Eds. H. Takagi and O. Boxma), 121-144, 1992.

[16] O. J Boxma, H. Levy, U Yechiali. Cyclic reservation schemes for efficient operation of multiple-queue single-server systems. Annals of Operations Research, 187-208, 1992.

[17] O. Boxma. Workloads and waiting times in single-server systems with multiple user classes. Queuing Systems, 5, 185-214, 1989.

[18] O.J. Boxma, W.P. Groenendijk. Pseudo-conservation laws in cyclic-service systems. Journal of Applied Probability, Vol. 24, No. 4, 949-964, Dec 1987.

[19] V. Kavitha and E. Altman. Continuous polling models and application to Ferry assisted WLAN. Under revision with Annals of Operations Research (Special issue on polling systems). Also, available at http://hal.inria.fr/inria-00573799/en.

[20] V. Kavitha. Continuous polling with rerouting: Performance and modeling of ferry assisted wireless LANs. Submitted to Elsevier Computer Networks (Special issue on Complex Dynamic Systems), 2011.

[21] V. Kavitha and E. Altman. Analysis and design of message ferry routes in sensor networks using polling models. WiOpt May 31-Jun 04, Avignon, France, 2010.

[22] V.Kavitha and E. Altman. Queueing in space: design of message ferry routes. 21st International Teletraffic Congress (ITC 21), 2009.

[23] W. Saad, Z. Han, T. Başar M. Debbah and A. Hjorungnes. A selfish approach to coalition formation among unmanned air vehicles. Gamenets, Istanbul, Turkey, 2009.

[24] W. Whitt. A review of $l=\lambda w$ and extensions. Queueing Systems: Theory and Applications, Volume 9 Issue 3, Oct. 1991. 